\title{
Epidural hematomas after the implantation of thoracic paddle spinal cord stimulators
}

\author{
Nazih A. Moufarrij, MD \\ Department of Surgery, University of Kansas, Wichita, Kansas
}

OBJECTIVE There is little information on the frequency of symptomatic epidural hematomas after the implantation of paddle spinal cord stimulators (SCSs) in the thoracic spine. The purpose of this paper is to provide this metric and compare it to the frequency of symptomatic epidural hematomas for all other thoracic laminectomies combined.

METHODS This study involved retrospectively analyzing the experience of a single surgeon in a consecutive series of patients who underwent the implantation of a thoracic paddle SCS with respect to the occurrence of a symptomatic epidural hematoma. For comparison, the occurrence of a symptomatic epidural hematoma in non-SCS thoracic laminectomies done during the same period of time was determined.

RESULTS One hundred fifty-four thoracic paddle SCSs were implanted between May 2002 and February 2015. Despite perfect hemostasis and no preoperative risk factors, 4 of 154 patients (2.60\%) developed postoperative lower-extremity weakness caused by an epidural hematoma. There were no other causes of a neurological deficit. In 3 of the 4 patients, the symptoms were delayed. Over the same time period, only 1 of 119 patients $(0.84 \%)$ developed a postoperative motor deficit from a symptomatic epidural hematoma after a non-SCS laminectomy.

CONCLUSIONS The occurrence of epidural hematomas after thoracic paddle SCS implantation may be underreported. Suggestions are given to decrease its incidence. It seems paradoxical that an epidural hematoma occurred 3 times more often after small SCS thoracic laminectomies than after larger non-SCS thoracic laminectomies. If confirmed by future studies, this finding may suggest that the intrusion of instruments into a confined epidural sublaminar space or the presence of a paddle and a hematoma in this restricted space may account for this differential.

http://thejns.org/doi/abs/10.3171/2015.8.JNS15396

KEY WORDS paddle spinal cord stimulator; thoracic epidural hematoma; frequency; paraparesis; paraplegia

$\mathrm{S}$ INCE its first use in 1967, the number of spinal cord stimulator (SCS) surgeries is expected to keep increasing due to the mounting numbers of patients with pain syndromes and the demonstration that SCS may help in pain control. ${ }^{5,11}$ The purpose of this paper is to report the frequency of the rare but catastrophic complication of symptomatic epidural hematoma after the implantation of a thoracic paddle SCS.

\section{Methods}

\section{Surgical Technique}

I performed all operations without resident involvement, which consisted of the insertion of a paddle electrode via a small laminectomy at the level of interest. Typically 0.5 to $1.5 \mathrm{~cm}$ of dura in the cephalocaudal direction and $1 \mathrm{~cm}$ of dura in the right to left direction is exposed. Hemostasis was secured methodically at each anatomical level going down to the epidural space then coming out from the epidural space to the skin. The passing of the paddle or any instrument between the dura and the lamina was done with utmost care.

\section{Clinical Data}

The clinical data were reviewed to identify the patients who developed any lower-extremity weakness after surgery. Pertinent information was extracted from their charts. Phone calls were then made to inquire about the events prior and subsequent to the motor deficit and ask about the recovery of motor function.

To compare the frequency of symptomatic epidural hematoma after laminectomy for SCS versus non-SCS, the clinical data for all patients undergoing a laminectomy for non-SCS, over the same period of time as this study, were reviewed to identify those who developed a symptomatic epidural hematoma. The non-SCS group included tumors, 
central stenosis, fractures, hematomas, infections, and herniated discs.

\section{Review of the Literature}

A PubMed literature search in all languages and with no date restriction was undertaken using the terms "epidural hematoma" and "paddle spinal cord stimulator." The bibliographies from the papers identified by PubMed were reviewed to find more applicable publications. Articles with percutaneous leads only were not used. When looking for incidence, several papers did not have the denominator available. Only 2 papers provided an incidence, ${ }^{3,11}$ while several were case reports. ${ }^{1,7,8,12}$

\section{Results}

\section{Symptomatic Epidural Hematomas in SCS and Non-SCS Thoracic Laminectomies}

One hundred fifty-four thoracic paddle SCSs were implanted between May 2002 and February 2, 2015. This represents the total number performed by me during my career until the closure of this study. Three patients developed severe paraparesis or paraplegia (1.95\%), and 1 patient had unilateral lower-extremity weakness $(0.65 \%)$ postoperatively. The incidence of this complication is $2.60 \%$ ( 4 of 154 patients). None of these 4 patients had any risk factors for bleeding, and their surgeries were uneventful. There were no other causes of spinal cord injuries. Over the same period, 119 patients underwent a laminectomy for non-SCS: 64 epidural metastases, 21 stenoses with or without disc herniations, 13 epidural infections, 7 fractures, 5 spontaneous epidural hematomas, 5 intradural meningiomas, 3 intramedullary metastases, and 1 intradural schwannoma. Only 1 of 119 patients $(0.84 \%)$ developed a postoperative motor deficit from a symptomatic epidural hematoma. Therefore, in my experience, SCS thoracic laminectomy resulted in 3 times more symptomatic epidural hematomas than non-SCS thoracic laminectomies ( $2.60 \%$ vs $0.84 \%$, respectively).

\section{First Severe Case of Paraparesis/Paraplegia}

The first complication occurred 8 years after I started performing this operation. A 70-year-old male patient developed marked bilateral lower-extremity weakness at 4.5 days after the implantation of a thoracic paddle SCS. The only movement in the lower extremities was in the toes, which was minimal. At surgery, hemostasis was perfect. The patient arrived to the hospital around 16 hours after the onset of his weakness. He was rushed to surgery without imaging, and an epidural hematoma was evacuated. He eventually improved and was able to walk 100 feet with a walker. The patient died 13 months after surgery of urosepsis, probably due to a neurogenic bladder from the epidural hematoma.

\section{Second Severe Case of Paraparesis/Paraplegia}

A 58-year-old female patient was brought to the hospital at 6 days after an uneventful implantation of a thoracic paddle SCS. She could move her toes only minimally. She was immediately taken to surgery without imaging. An epidural hematoma was evacuated. She improved and was able to walk independently. Subsequently, she requested reimplantation of a paddle SCS. This was done and she did well, only to die suddenly 3 days later, probably due to a cardiac cause as she had preexisting heart disease.

\section{Third Case of Paraparesis/Paraplegia}

A 59-year-old female patient was brought back to the hospital because she was paraplegic at 2 days after the implantation of a paddle SCS. She had previously been implanted with a paddle SCS by another surgeon that was removed due to infection. After the first implantation, she developed reversible unilateral leg weakness. Her deficit, after the second implantation, started 21 hours prior to her readmission, at which time her systolic blood pressure was $213 \mathrm{~mm} \mathrm{Hg}$. She was in pain and did not take her pain and antihypertensive medications due to nausea. When examined, she had no movement and no sensation in her lower extremities. At surgery, an epidural hematoma was evacuated. Three days later, MRI showed a markedly swollen cord without any residual compression. At 5 months postoperatively, she had minimal movement in her lower extremities and was wheelchair bound.

\section{A Case of Unilateral Leg Weakness}

A 58-year-old female patient was taken back to surgery the day following her paddle SCS implantation because of stimulation failure and the new onset of unilateral leg weakness. An epidural hematoma was evacuated ipsilateral to the weakness and the paddle was removed. Two years later, she still uses a cane to walk. She was walking normally prior to SCS implantation.

\section{Discussion}

\section{Prevention Strategy: Surgical Technique and Communication With the Patient}

It is important to note that epidural hematoma occurred despite normal coagulation, perfect hemostasis, and an experienced surgeon who had been doing this operation for 8 years at the time of the first complication. While those elements are important, they are not sufficient to prevent this complication. . $^{1,9,13}$ Their presence should not give the surgeon a false sense of security, especially since the thoracic spinal cord has less blood flow than the cervical or lumbar cords, ${ }^{6}$ thereby making it more vulnerable to compressive ischemia. Furthermore, an epidural hematoma could be about 8 times more frequent after a thoracic laminectomy than after a cervical or a lumbar laminectomy. ${ }^{2}$

Given the above, it is crucial to talk to the patient before surgery about this complication and establish a personal rapport so that he/she feels that the two together (patient and physician) will do everything possible to avoid this complication. In particular, it is not enough to hand patients instruction papers because they may not read, understand, or act on them. The surgeon should make sure that the patient and a family member or friend know the early symptoms of cord compression, realize its extreme emergency, and know what should be avoided such as skipping antihypertensive medications (due to nausea as in one of the patients in this study), taking medications with a blood-thinning effect, excessive coughing, holding 
a sneeze, and vigorous activities with the arms. In addition to this information, a reliable phone number should be written in the dismissal instructions. In fact, I give my patients my personal mobile number to stress the importance of this issue and to avoid delays by intermediaries. Dismissals should not be hurried, making sure that pain, nausea, and hypertension are well controlled.

\section{Surgical Treatment}

Because every minute counts, time should not be wasted performing imaging studies that will not change the management. ${ }^{4,10}$ The patient is taken to the operating room and the paddle is removed. If a hematoma is found, it should be evacuated by extending the laminectomy. If there is no hematoma, extension of the laminectomy would have been done to ascertain that this indeed is the case. Decompression of the cord might help in the event of central canal stenosis (compounded by the paddle) or cord contusion.

\section{Is There a Higher Incidence in Comparison With Non-SCS Thoracic Laminectomies?}

In paddle SCS placement, the dura over the spinal cord is usually exposed over an area of about 0.5 to $1.5 \mathrm{~cm}^{2}$. In all other thoracic laminectomies, the dura is exposed over larger areas for pathologies such as tumors, central canal stenosis, fractures, hematomas, epidural abscesses, and disc herniations. During the same time period of this study, I performed 119 non-SCS thoracic laminectomies with only 1 symptomatic postoperative epidural hematoma in a patient who underwent surgery for an epidural abscess. The incidence of epidural hematoma in non-SCS procedures is $0.84 \%$, which is about 3 times less than in a laminectomy for SCS. This 3-fold increase in the occurrence of an epidural hematoma when comparing small SCS thoracic laminectomies with usually much larger SCS thoracic laminectomies seems paradoxical. To our knowledge, other surgeons have not mentioned this comparison between thoracic epidural hematomas in SCS and non-SCS laminectomies. It will be interesting to see if this finding is confirmed by other studies. If it is, this would suggest that the higher incidence of epidural hematoma in paddle SCS implantation may be related to the insertion of instruments and/or the presence of the paddle in the epidural sublaminar space. In fact, judging by the area of dura exposed and the extent of the procedure, one would have expected the opposite: non-SCS laminectomies to have a higher incidence of epidural hematomas. The paddle may open the potential epidural space ever so little as to allow the entry of blood between the dura and the overlying lamina. The insertion of a paddle or instruments under the lamina may cause weakening in or injury to the vascular wall with rupture when the patient coughs, sneezes, or has a hypertensive peak. It may well be that the presence of lamina in the non-SCS group confines the hematoma to a smaller space, thereby making symptomatic cord compression more likely.

\section{Is Symptomatic Epidural Hematoma an Underreported Complication?}

The $2.60 \%$ rate of symptomatic epidural hematoma in paddle SCS in this series is the highest reported in the lit- erature. This could be an unfortunate coincidence for this group of patients. However, there is some evidence to suggest that this complication may be underreported. ${ }^{2,11,16}$ It also seems that it has not received the attention it deserves as suggested by the comprehensive paper of Levy et al. ${ }^{11}$ One of the published comments on their paper states that the $0.6 \%$ risk of spinal cord injury from all causes (epidural hematomas and other causes) after paddle implantation is likely an underestimate. By way of comparison, the stroke rate after carotid endarterectomy and the rate of a neurological deficit after pedicle screw implantation have been much more scrutinized. It may be worthwhile for professional societies to send a questionnaire regarding this complication to surgeons who implant paddle SCSs.

\section{Delayed Occurrence}

Three of the 4 complications-also by far the most severe-occurred after the patient left the hospital, with the latest being at 6 days later. This finding was reported by others, ${ }^{2,11,14,15}$ underscoring the importance of educating the patient to stay vigilant especially for the first 2 weeks after surgery until epidural scarring sets in.

\section{Estimates of the Number of Implants and Symptomatic Epidural Hematomas}

Knowing that in a 3-year period between September 1, 2007, and August 31, 2010, 44,587 paddle SCSs were implanted in the US, ${ }^{11}$ and that for the year 2013 about 50,000 percutaneous and paddle SCSs were implanted in the US, ${ }^{16}$ would suggest that about $30 \%$ of those are paddle SCSs. Assuming an incidence of symptomatic epidural hematoma of $1 \%{ }^{16}$ would imply that 150 patients became paraparetic or paraplegic that year after paddle SCS implantation in the US.

\section{Conclusions}

The incidence of lower-extremity weakness due to a postoperative epidural hematoma was $2.60 \%$ in this series, despite normal coagulation and perfect hemostasis. In 3 of the 4 patients it was delayed. This complication may be underreported. Every effort should be made by individual surgeons and professional societies to decrease this incidence. The 3 -fold increase in the frequency of an epidural hematoma when comparing small SCS thoracic laminectomies with much larger non-SCS thoracic laminectomies seems paradoxical. It could be due to the intrusion of instruments into a confined epidural sublaminar space or the presence of a paddle and a hematoma in this restricted space.

\section{References}

1. Amrani J: Epidural hematoma following implantation of a permanent spinal cord stimulator paddle. Neuromodulation 17:279-281, 2014

2. Aono H, Ohwada T, Hosono N, Tobimatsu H, Ariga K, Fuji $\mathrm{T}$, et al: Incidence of postoperative symptomatic epidural hematoma in spinal decompression surgery. J Neurosurg Spine 15:202-205, 2011

3. Barolat G: Experience with 509 plate electrodes implanted epidurally from $\mathrm{C} 1$ to L1. Stereotact Funct Neurosurg 61:60-79, 1993 
4. Cabana F, Pointillart V, Vital J, Sénégas J: [Postoperative compressive spinal epidural hematomas. 15 cases and a review of the literature.] Rev Chir Orthop Repar Appar Mot 86:335-345, 2000 (Fr)

5. Cameron T: Safety and efficacy of spinal cord stimulation for the treatment of chronic pain: a 20-year literature review. J Neurosurg 100 (3 Suppl Spine):254-267, 2004

6. Dommisse GF: The blood supply of the spinal cord. A critical vascular zone in spinal surgery. J Bone Joint Surg Br 56:225-235, 1974

7. Franzini A, Ferroli P, Marras C, Broggi G: Huge epidural hematoma after surgery for spinal cord stimulation. Acta Neurochir (Wien) 147:565-567, 2005

8. Kebaish KM, Awad JN: Spinal epidural hematoma causing acute cauda equina syndrome. Neurosurg Focus 16(6):e1, 2004

9. Kou J, Fischgrund J, Biddinger A, Herkowitz H: Risk factors for spinal epidural hematoma after spinal surgery. Spine (Phila Pa 1976) 27:1670-1673, 2002

10. Lawton MT, Porter RW, Heiserman JE, Jacobowitz R, Sonntag VK, Dickman CA: Surgical management of spinal epidural hematoma: relationship between surgical timing and neurological outcome. J Neurosurg 83:1-7, 1995

11. Levy R, Henderson J, Slavin K, Simpson BA, Barolat G, Shipley J, et al: Incidence and avoidance of neurologic complications with paddle type spinal cord stimulation leads. Neuromodulation 14:412-422, 2011

12. Meyer SC, Swartz K, Johnson JP: Quadriparesis and spinal cord stimulation: case report. Spine (Phila Pa 1976) 32:E565-E568, 2007

13. Santiago FM, Santiago J, Prieto M, García-Sánchez MJ, Sánchez-Carríon JM, Martínez-Tellería A, et al: [Dorsal epidural hematoma after implantation of a dorsal nerve stimulator.] Rev Esp Anestesiol Reanim 52:440-441, 2005 (Span)
14. Smith CC, Lin JL, Shokat M, Dosanjh SS, Casthely D: A report of paraparesis following spinal cord stimulator trial, implantation and revision. Pain Physician 13:357-363, 2010

15. Uribe J, Moza K, Jimenez O, Green B, Levi AD: Delayed postoperative spinal epidural hematomas. Spine J 3:125-129, 2003

16. Walker J: When spine implants cause paralysis, who is to blame? Wall Street Journal. April 15, 2014. (http://www. wsj.com/articles/SB1000142405270230451250457949384364 7492538) [Accessed December 22, 2015]

\section{Disclosures}

The author reports no conflict of interest concerning the materials or methods used in this study or the findings specified in this paper.

\section{Supplemental Information}

\section{Previous Presentations}

Portions of this work were presented in poster form at the 81st American Association of Neurological Surgeons Annual Scientific Meeting, New Orleans, Louisiana, April 27 to May 1, 2013, and in abstract form at the 3rd International Conference on Neurology and Epidemiology, Abu Dhabi, November 21-23, 2013.

\section{Correspondence}

Nazih Moufarrij, Department of Surgery, University of Kansas, 8814 Windwood Cir., Wichita, KS 67226. email: n_moufarrij@ hotmail.com. 